\title{
Changes in islet blood flow in rats with NIDDM
}

\author{
N. Atef, B.Portha, L. Pénicaud \\ Laboratoire de Physiopathologie de la Nutrition, CNRS URA 307, Université Paris VII, Paris, France
}

Summary Islet blood flow was quantified in NIDDM rats either of the GK strain on after neonatal injection of STZ (n0-STZ), using the non-radioactive microsphere technique. In the basal state, there was a good correlation between plasma insulin level and islet blood flow, i. e. both were increased or decreased in comparison to those of control rats in GK and n0-STZ rats, respectively. The increased islet blood flow and plasma insulin levels observed in the GK rats were abolished by bilateral subdiaphragmatic vagotomy.
During a glucose challenge, whereas plasma insulin and islet blood flow were doubled in control rats, these parameters were not modified in the diabetic rats. These data demonstrate an alteration in the islet blood flow of diabetic rats during a glucose challenge which could participate in the abnormal glucose-induced insulin secretion previously described in these two models. [Diabetologia (1994) 37: 677-680]

Key words GK rats, n0-STZ, insulin, islet blood flow.
Diabetes mellitus is known to be associated with profound changes in glucose metabolism. Indeed, animals with NIDDM exhibit a glucose intolerance, reflecting a decreased ability to release insulin in response to glucose $[1,2]$. It has also been demonstrated that diabetes induces profound alterations in haemorheological parameters [3]. On the other hand, numerous data have shown that islet blood flow increases concomitantly with insulin. Thus, in normal rats, an enhanced plasma glucose concentration consistently leads to an enhancement of both islet blood flow and insulin level [46]. Furthermore, in obese hyperinsulinaemic rats, islet blood flow is increased under basal conditions and an injection of glucose induces a higher than normal islet blood flow and insulin secretion [7]. On the basis of these data, our aim was to determine if islet blood flow could be altered in rats presenting NIDDM. We used

Received: 27 December 1993

and in revised form: 28 February 1994

Corresponding author: Dr. L.Pénicaud, Laboratoire de Physiopathologie de la Nutrition, CNRS URA 307, Université Paris VII, Tour 23-33, 2 place Jussieu, F-75251 Paris Cedex 05, France

Abbreviations: NIDDM, non-insulin-dependent diabetes mellitus; STZ, streptozotocin. two different models, one being the neonatally STZtreated rat $[1,2,8]$ which presents decreased plasma insulin levels both under basal and glucose-stimulated conditions. The second is a genetic model, the GK rat [9] characterized by an enhanced basal plasma insulin concentration but a decreased secretory capacity in response to glucose $[10,11]$. In the present study we determined islet blood flow in these two models of diabetes, before and after glucose administration.

\section{Materials and methods}

Animals: Rats used in this study were female weighing between 200 and $220 \mathrm{~g}$. They were fed ad libitum on standard laboratory chow (UAR, Villemoisson, France) and maintained in an animal quarter in which the temperature was kept at $24^{\circ} \mathrm{C}$ with light on from 07.00 to 19.00 hours. Insulin deficiency was induced in neonates raised from Wistar rats, by intraperitoneal administration of STZ $(100 \mathrm{mg} / \mathrm{kg})$ dissolved in $30 \mu \mathrm{l}$ of citrate buffer $(0.05 \mathrm{~mol} / \mathrm{l}, \mathrm{pH} 4.5)$. Spontaneous development of neonatal diabetes (n0-STZ) led to NIDDM in the adult [8]. The GK rats were bred in our laboratory. The spontaneous diabetic state was produced by selective breeding repeated over numerous generations with glucose intolerance as selection index and starting from a non-diabetic Wistar colony. The diabetic state become stable after F30 [9]. The GK rat colony in our laboratory started with progenitors issued from F35 of the Japanese colony. Female Wistar rats were used as controls. 
Blood flow measurements: Blood flow measurements were performed as described by Jansson et al. [4, 5, 12]. This procedure makes the exocrine part of the pancreas transparent so that both islets and microspheres can be seen, thus allowing the counting of the latter both in the acinar and exocrine tissues. This is achieved by freezing and thawing of small pieces of the pancreas and examination in transmitted light using a microscope equipped with both dark and bright field illumination.

After a 4-h fast, rats were anaesthetized with sodium pentobarbital $(0.8 \mathrm{ml} / \mathrm{kg}$, i.p. $)$ and heparinized with an i.v. injection of 200 IU of heparin (Roche, Neuilly, France). Polyethylene catheters were placed into the lower abdominal aorta, via the left femoral artery and into the left ventricle of the heart, via the right carotid artery.

Non-radioactive microspheres $\left(1-1.5 \times 10^{5}\right)$ (New England Nuclear Corp., Boston, Mass., USA) with a 10-um diameter were injected via the intracardiac catheter. Simultaneously an arterial blood sample was withdrawn from the catheter in the abdominal aorta with a peristaltic pump adjusted to a rate of $0.6 \mathrm{ml} / \mathrm{min}$ for $90 \mathrm{~s}$. This reference sample was used to calculate the organ blood flow as described previously $[4,5,12]$. Then $500 \mu \mathrm{l}$ of blood were quickly sampled, centrifuged and the plasma frozen. Plasma glucose and insulin concentrations were determined using respectively, a glucose analyser (Glucose analyzer 2, Beckman, Fullerton, Calif., USA), and a radioimmunoassay kit (INSIK 1; CEA, Saclay, France).

The rats were killed by cervical dislocation and the pancreas and both adrenal glands were removed, blotted and weighed. The organs and the reference sample were further processed and examined for microsphere content, as previously described [4, 5]. A minimum number of 150 microspheres were counted in each rat as recommended by Jansson et al. [4]. The microsphere content of the adrenal gland was used as a measure of the mixing of the microspheres with the blood. A difference of more than $10 \%$ between the two glands excluded the animal from the study. Likewise, blood pressure and body temperature were continuously monitored and variations in blood pressure or in body temperature exceeding $10 \%$ and $0.5^{\circ} \mathrm{C}$, respectively led to the exclusion of the animal.

In other rats, the left saphenous vein was dissected free and used for injection of glucose $\left(37.5 \mathrm{mg} \cdot 500 \mu 1^{-1} \cdot 100 \mathrm{~g}^{-1}\right), 5 \mathrm{~min}$ before the blood flow measurement.

In a third group of Wistar and GK rats, a bilateral subdiaphragmatic vagotomy was performed $30 \mathrm{~min}$ before the injection of microspheres. A laparotomy was performed and the two branches of the vagus along the oesophagus were cut just under the diaphragm. The incision was then sutured.

\section{Statistical analysis}

Results are expressed as mean \pm SEM. Statistical significance of differences between means were evaluated using the Mann Whitney U test.

\section{Results}

\section{Islet blood flow in the basal state}

$G K$ rats: In the basal state, both plasma glucose and insulin concentrations were higher in GK than in control rats (Fig. 1). Concomitantly to these increases, islet blood flow was higher in GK rats (Fig. 1).
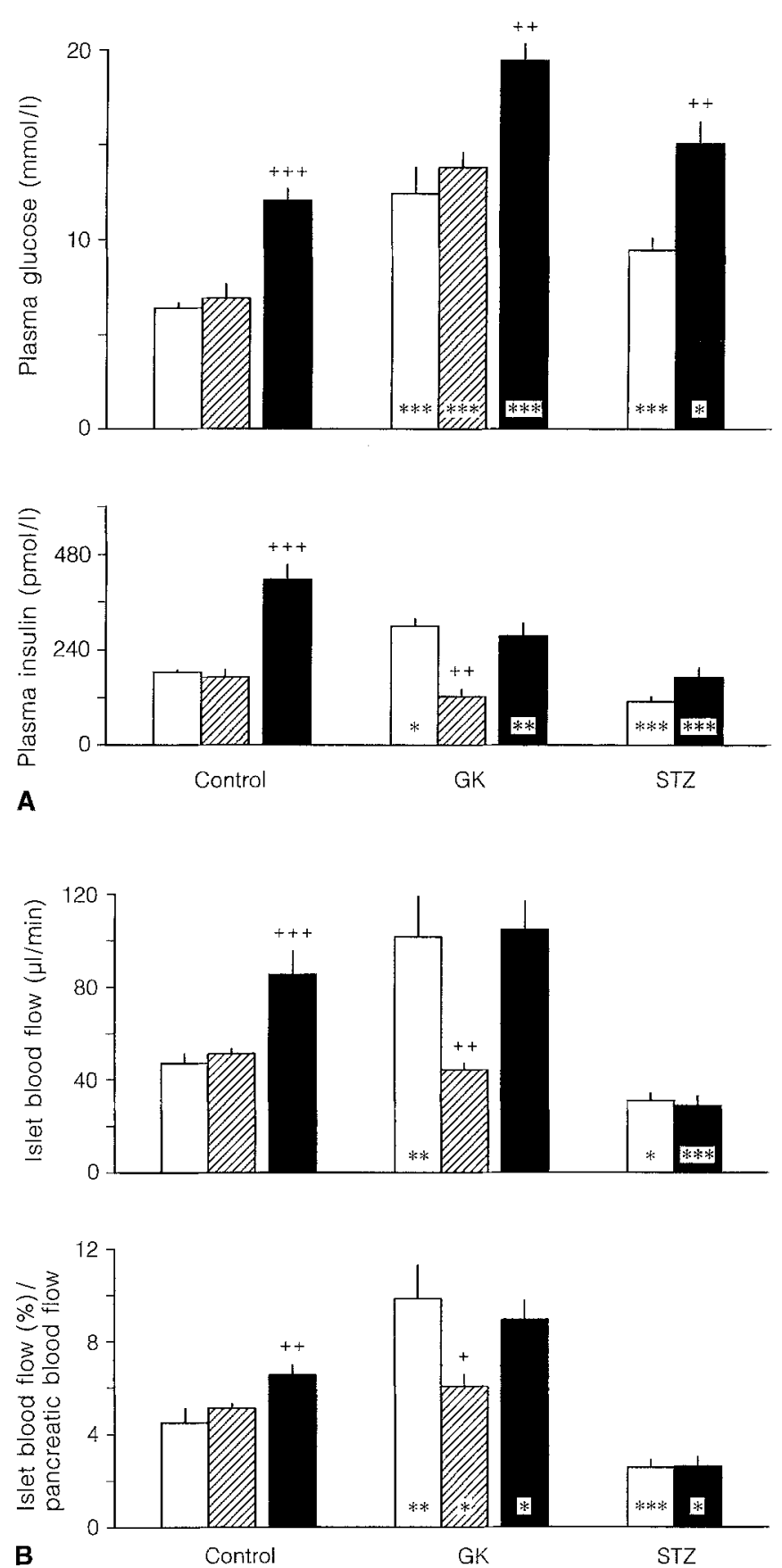

Fig.1. (A) Plasma glucose and insulin concentrations, $(\mathbf{B})$ islet blood flow and percent of islet blood flow from pancreatic blood flow in intact control, GK and n0-STZ rats $(\square)$ or vagotomized control and GK rats $(\mathbb{Z})$ under basal condition or in intact rats after an i.v. glucose load $(37.5 \mathrm{mg} / 100 \mathrm{~g}$; ). Data are means \pm SEM of four to seven determinations. Statistically significant difference: between control and diabetic rats $* p<0.05$; $* * p<0.01$; *** $p<0.001$; between intact and vagotomized or basal and glucose injected conditions $++p<0.01$; $+++p<0.001$

n0-STZ rats: In the adult rats treated neonatally with STZ, plasma glucose was significantly higher than in controls (Fig. 1). By contrast, plasma insulin and islet blood flow were two times in n0-STZ than in control rats (Fig. 1). This decrease of islet blood flow was even 


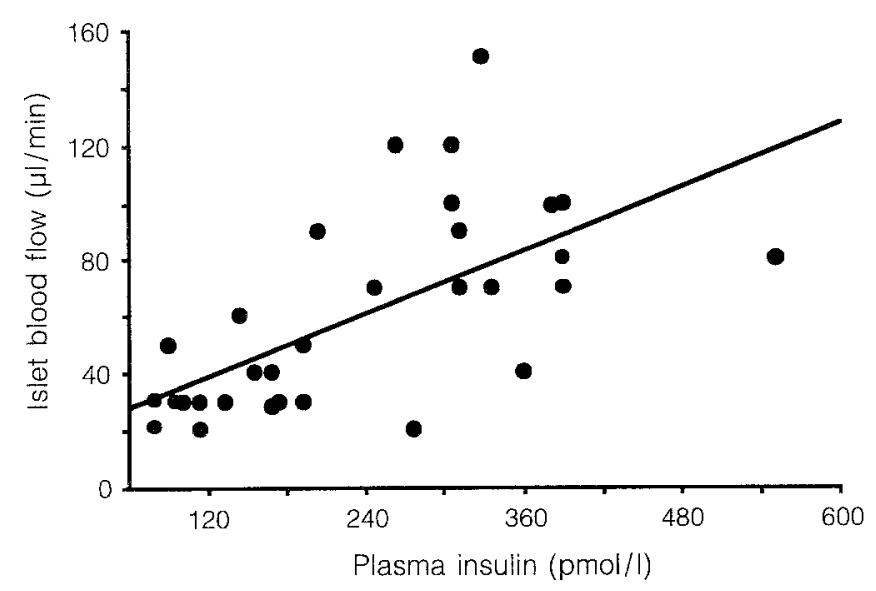

Fig. 2. Islet blood flow plotted against the plasma insulin concentration for all animals. The equation of the line is $y=1.11 \times+17.01(r=0.638 ; n=29 ; p<0.001)$

more apparent when we expressed islet blood flow as a percent of the total pancreatic blood flow since this parameter (Fig. 1) was slightly but not significantly higher in $\mathrm{n} 0-\mathrm{STZ}\left(1.5 \pm 0.3 \mathrm{ml} \cdot \mathrm{min}^{-1} \cdot \mathrm{g}^{-1}\right)$ than in control rats $\left(1.1 \pm 0.1 \mathrm{ml} \cdot \mathrm{min}^{-1} \cdot \mathrm{g}^{-1}\right)$.

Effect of vagotomy in the Wistar and GK rats. In Wistar rats vagotomy did not change either plasma glucose and insulin levels, or pancreatic and islet blood flow (Fig.1). In GK rats, section of the vagus nerve induced a significant decrese of plasma insulin which returned to the level of control rats, without modification of plasma glucose (Fig. 1). Vagotomy reduced islet blood flow by a factor of two in GK rats (Fig. 1). As a consequence, islet blood flow was no different in vagotomized GK rats and in intact controls.

Islet blood flow after glucose administration. The glucose injection induced a significant increase in plasma glucose in controls as well as in n0-STZ and GK rats (Fig. 1). In control rats, glucose administration led to a two-fold increase in both the plasma insulin level and islet blood flow (Fig. 1). By contrast, the same injection affected neither plasma insulin concentration nor islet blood flow in either group of diabetic rats (Fig. 1).

Correlation. When the values of all the groups were pooled, plasma insulin was positively correlated with islet blood flow $(r=0.64, F=19.9, p<0.001)$ (Fig. 2).

\section{Discussion}

The data show that islet blood flow is altered in NIDDM rats either of the GK strain or after neonatal injection of STZ. This alteration paralleled that found in the plasma insulin concentration. This is supported by the highly significant correlation between islet blood flow and plasma insulin found in this study and which has been demonstrated previously [5-7]. However, this relationship between insulin levels and islet blood flow has been questioned in other studies which show no link between insulin secretion and glucose stimulated islet blood flow in rats during starvation [13], after partial pancreatectomy [14] or after islet grafts [15]. This discrepancy could be due to the fact that in these latter studies [13-15] which were performed shortly after the treatment, the increased islet blood flow may be associated with the stimulation of growth of the islet organ and/or with acute changes of islet metabolism, whereas our study and others [5-7] reflect adaptative changes.

In the basal state, both plasma insulin and islet blood flow were higher and lower, respectively in GK rats and n0-STZ rats than in controls. Furthermore, whereas after glucose administration, plasma insulin and islet blood flow were almost doubled in control rats, the same injection did not change these parameters in the two models of diabetic rats. The inability of glucose to induce an increase in islet blood flow appears to be one of the specific characteristics of the diabetic rats. This is reminiscent of what has been demonstrated in rats unable to increase their islet blood flow in response to glucose after partial pancreatectomy [14].

It is noteworthy that in the basal state, GK rats presented, as observed in genetic or experimentally-induced obese rats, an increased plasma insulin concentration together with a higher islet bloot flow [7]. This suggests a common mechanism which could be an alteration in the control exerted by the central nervous system on these parameters $[6,7]$. Such an explanation is further supported by the observation that whereas vagotomy did not change any parameters in control rats, it totally abolished the enhanced plasma insulin and islet blood flow observed in GK rats. These results confirm the data of Svensson et al. [16] showing heightened blood flow of the F1-hybrids of the GK-Wistar rat and its normalization after vagotomy. This is similar to observations described in obese Zucker rats [7].

The mechanisms underlying these alterations of islet blood flow in diabetic rats are currently unknown. A direct effect of STZ on islet blood flow has been ruled out by previous investigation [17]. The alterations could be due to the deficient metabolism of glucose in the islets of the diabetic rats [18] which may produce low concentrations of glucose metabolites, e.g. lactate, which are known to affect the microcirculation [19]. However, this is unlikely since it has previously been demonstrated that the glucose-induced increase in islet blood flow is independent of the metabolism of glucose in the islets [13]. It could also be due to hyperglycaemia per se which has been shown to increase islet blood flow [20]; however, both n0-STZ and GK rats were hyperglycaemic but presented a decrease and an increase in islet blood flow, respectively. Another explanation could be that the activity of the central nervous system, which is known to mediate the glucose-induced increase in islet blood flow [6] as well as the in- 
crease in islet blood flow observed in the obese rat [7], has been modified. This modification could be directly due either to an effect of STZ or to a genetic mutation on the neurons containing glucoreceptors. It could also be due to the long-standing glucose intolerance which has been registered in these two models $[2,9,10]$.

In summary these data demonstrate that, in NIDDM rats, a decreased glucose-induced stimulation of islet blood flow is present. It could participate in the defect in insulin secretion described in these rats.

Acknowledgements. We would like to thank Ms. D. Bailbé for her expert technical assistance, Dr. A. Ktorza for his careful reviewing of this manuscript and Dr. L. Jansson for his help and advice in setting the microsphere technique. This work was partly supported by a grant from the Institut National de la Santé et de la Recherche Médicale (CRE 910310) and connected to " $\beta$ cell transplant", European Concerted Action for the Treatment of Diabetes.

\section{References}

1. Giroix MH, Portha B, Kergoat M, Picon L (1983) Glucose insensitivity and amino acid hypersensitivity of insulin release in rats with non insulin dependent diabetes. A study with the perfused pancreas. Diabetes 32: 445-451

2. Portha B, Blondel O, Serradas P, et al. (1989) The rats models of non insulin-dependent diabetes induced by neonatal streptozotocin. Diab Metab 15: 61-75

3. McMillan DE (1984) The microcirculation in diabetes. Microcirc Endothelium Lymphatics 1: 3-24

4. Jansson L (1984) The blood flow to the pancreas and the islets of Langerhans during an intraperitoneal glucose load in the rat. Diabetes Res 1: 111-114

5. Jansson L, Hellerström C (1983) Stimulation by glucose of the blood flow to the pancreatic islets of the rats. Diabetologia $25: 45-50$

6. Jansson L, Hellerström C (1986) Glucose induced changes in pancreatic islet blood flow mediated by central nervous system. Am J Physiol 251: E644-E647

7. Atef N, Ktorza A, Picon L, Pénicaud L (1992) Increased islet blood flow in obese rats: role of the autonomic nervous system. Am J Physiol 262: E736-E740

8. Blondel O, Bailbe D, Portha B (1989) Relation of insulin deficiency to impaired insulin action in NIDDM adult rats given streptozotocin as neonates. Diabetes 36: 610-617
9. Goto Y, Suzuki K, Sasaki M, Ono T, Abe S (1988) GK rats as model of non-insulin-dependent diabetes. Selective breeding over 35 generations. In: Shafrir E, Renold AE (eds) Frontiers in diabetes research. Lessons from animal diabetes II. Libbey London, pp 301-303

10. Portha B, Serradas P, Bailbé D, Suzuki K, Goto Y, Giroix MH (1991) B cell insensitivity to glucose in the GK rats, a spontaneous nonobese model for type II diabetes. Diabetes 40: 486-491

11. Giroix MH, Vesco L, Portha B (1992) Functional and metabolic perturbations in isolated pancreatic islets from the GK rat, a genetic model of non insulin dependent diabetes. Endocrinol 132: 815-822

12. Jansson L, Hellerström C (1981) A rapid method of visualizing the pancreatic islets of studies of islet capillary blood flow using non radioactive microspheres. Acta Physiol Scand 113: 371-374

13. Jansson L (1985) Dissociation between pancreatic islet blood flow and insulin release in the rat. Acta Physiol Scand 124: 223-228

14. Jansson L, Sandler S (1991) Partial pancreatectomy in rats causes an impairment of the glucose induced stimulation of pancreatic islet blood flow. Experientia 47:627-629

15. Sandler S, Jansson L (1987) Blood flow measurements in autotransplanted pancreatic islets of the rat. J Clin Invest 80: $17-21$

16. Svensson AM, Abdel-Halim SM, Efendic S, Jansson L, Bodin B, Östenson C-G (1993) F1-hybrids of the GK-Wistar rat have a nervously mediated increased islet blood perfusion. Diabetologia 36 [Suppl 1]: A86 (Abstract)

17. Jansson L, Sandler S (1992) Alloxan but not streptozotocin, increases blood perfusion of pancreatic islets in rats. Am J Physiol 263: E57-E63

18. Portha B, Kergoat M (1985) Dynamics of glucose-induced insulin release during the spontaneous remission of streptozotocin diabetes induced in newborn rat. Diabetes 34: 574 579

19. Haddy FJ, Scott JB (1968) Metabolically linked vasoactive chemicals in local regulation of blood flow. Physiol Rev 48: 688-707

20. Styrud J, Eriksson UJ, Jansson L (1992) A continuous 48hour glucose infusion in rats causes both an acute and a persistent redistribution of the blood flow within the pancreas. Endocrinol 130: 2692-2696 